FINAL TECHNICAL REPORT

\title{
IMPACTS OF BIOFILM FORMATION ON CELLULOSE FERMENTATION
}

\author{
Grant No. DE-FG02-02ER15330
}

\author{
Susan Leschine, University of Massachusetts
}

This report covers research performed during the project period, from September 1, 2002 through August 31, 2006.

Our research dealt with three major areas of investigation: i) characterization of formation of Cellulomonas uda biofilms on cellulose; ii) characterization of Clostridium phytofermentans biofilm development; colonization of cellulose and its regulation; iii) characterization of Thermobifida fusca biofilm development; colonization of cellulose and its regulation; and iii) description of the architecture of mature $C$. uda, $C$. phytofermentans, and $T$. fusca biofilms. This research is aimed at advancing understanding of biofilm formation and other complex processes involved in the degradation of the abundant cellulosic biomass, and the biology of the microbes involved. Information obtained from these studies is invaluable in the development of practical applications, such as the single-step bioconversion of cellulose-containing residues to fuels and other bioproducts.

Our results have clearly shown that these microbes rapidly colonize cellulose and form complex structures typical of biofilms. Furthermore, our observations suggest that, as cells multiply on nutritive surfaces during biofilms formation, dramatic cell morphological changes occur. We speculated that morphological changes, which involve a transition from rod-shaped cells to more rounded forms, might be more apparent in a filamentous microbe. In order to test this hypothesis, we initiated a study of biofilm formation by Thermobifida fusca, a thermophilic cellulolytic actinomycete commonly found in compost. The cellulase system of $T$. fusca has been extensively detailed through the work of David Wilson and colleagues at Cornell, and also, genome sequence of a $T$. fusca strain has been determine by the DOE Joint Genome Institute. Thus, $T$. fusca is an excellent subject for studies of biofilm development and its potential impacts on cellulose degradation.

Also, we completed a study of the chitinase system of $C$. uda. This work provided essential background information for understanding how $C$. uda degrades insoluble substrates. Additionally, we characterized biofilm formation by C. uda. Results of the first study mentioned above have been published, and a manuscript describing C. uda biofilm formation has been submitted for publication.

\section{Major accomplishments of the project include:}

- Development of media containing dialysis tubing (described by the manufacturer as "regenerated cellulose") as sole carbon and energy source and a nutritive surface for 
the growth of cellulolytic bacteria, and development of various microscopic methods to image biofilms on dialysis tubing.

- Demonstration that cultures of C. phytofermentans, an obligate anaerobe, C. uda, a facultative aerobe, and $T$. fusca, a filamentous aerobe, formed microbial communities on the surface of dialysis tubing, which possessed architectural features and functional characteristics typical of biofilms.

- Demonstration that biofilm formation on the nutritive surface, cellulose, involves a complex developmental processes, including colonization of dialysis tubing, formation of cell clusters attached to the nutritive surface, cell morphological changes, formation of complex structures embedded in extracellular polymeric matrices, and dispersal of biofilm communities as the nutritive surface is degraded.

- Determination of surface specificity and regulatory aspects of biofilm formation by $C$. phytofermentans, C. uda, and T. fusca.

- Demonstration that biofilm formation by T. fusca forms an integral part of the life cycle of this filamentous cellulolytic bacterium, including studies on the role of mycelial pellet formation in the $T$. fusca life cycle and a comparison of mycelial pellets to surface-attached $T$. fusca biofilms.

- Characterization of T. fusca biofilm EPS, including demonstration of a functional role for EPS constituents.

- Correlation of T. fusca developmental life cycle and cellulase gene expression.

\section{Results of our investigations are described below.}

\section{Formation of $C$. uda biofilms}

Prior to the start of the project, we observed that $C$. uda cells cultured in nitrogendepleted media were present as large aggregates. Addition of a nitrogen source (e.g., $\mathrm{NH}_{4} \mathrm{Cl}$, urea, $\mathrm{N}$-acetyl-D-glucosamine) to the growth medium resulted in a rapid dispersal of cells from these aggregates. C. uda cells attached tightly to cellulose and chitin, but did not colonize abiotic surfaces such as plastic or the glass surfaces of culture vessels. Under conditions of nitrogen limitation, cells appeared to form biofilms on nutritive surfaces including squid pen chitin and various forms of cellulose.

Cell aggregation and biofilm formation coincided with the production of extensive extracellular material. The appearance of $C$. uda cells stained with ruthenium red and examined by transmission electron microscopy suggested that cells grown under conditions of nitrogen depletion were embedded in a thick carbohydrate layer. This layer was absent from cells cultured in media containing sufficient available nitrogen. These results suggest that, under conditions of nitrogen limitation, C. uda cells adhere to nutritive substrates and develop into biofilm structures encased in an extracellular matrix of polysaccharide (EPS).

Scanning electron microscopic observations of $C$. uda biofilms on squid pen chitin revealed a typical biofilm architecture. Colonization of squid pens by $C$. uda cells resulted in a biofilm of growth that varied in density across the surface with patches of cell aggregates forming three-dimensional structures separated by open areas. These 
structures were similar to those observed in other biofilms and described as "pillars" or "mushrooms," separated by "channels." Chitinase activity was detected in supernatant fluids of biofilm-forming cultures, but cell-associated activity was not detected. This result suggests that cells in these biofilms may not degrade the surface to which they are directly attached. C. uda cells did not form biofilms when cultured on colloidal chitin. Cells grew as microcolonies on the surface of colloidal chitin particles, but detached as chitin was degraded. These results suggest that, as the readily degradable colloidal chitin was decomposed, sufficient nitrogen became available to signal a switch to planktonic growth.

Biofilm formation by C. uda was especially apparent when cellulose served as carbon source. Biofilms formed on cellulose only under conditions of nitrogen limitation, and addition of a nitrogen source to cultures resulted in a rapid dispersal of the cells from cellulose fibers. Cells were present on the surface of cellulose fibers (filter paper fibers) as patches of cell aggregates that appeared to form structures similar to those that formed on squid pen, described above. The filter paper appeared to be degraded by these biofilms, based on visual observations. Also, when C. uda was cultured with cellulose bound to Remazol Brilliant Blue 5R ("Cellulose-Azure," Sigma) under conditions of biofilm formation, dye was rapidly released from the substrate as measured by an increase in the optical density of culture supernatants measured at 595 $\mathrm{nm}$. C. uda biofilms were also grown on dialysis tubing. These biofilms were visualized by light microscopy, confocal scanning laser microscopy (CSLM), and scanning electron microscopy (SEM), and found to have architectural features similar to those described above and typical of single-species biofilms. C. uda biofilms degraded the dialysis tubing through a defined sequence of alterations in the structure of the dialysis tubing, observed both microscopically and macroscopically, that resulted in its complete disintegration.

Our results demonstrated the involvement of biofilms in the degradation of natural polymers by $C$. uda and indicated that biofilm formation on biological surfaces may play a nutritive role for the microbe. Colonization of natural polymers and biofilm formation may confer on $C$. uda cells a selective advantage over other cellulolytic and/or chitinolytic microorganisms, allowing the bacteria to "secure" a nutrient in a nutrient-poor environment where competition for food is high.

\section{Formation of biofilms by C. phytofermentans}

Previous work of our laboratory demonstrated that $C$. phytofermentans rapidly degrades cellulose and produces exceptionally large amounts of ethanol as a fermentation product. This unusual fermentation pattern indicated that $C$. phytofermentans might be useful in studies of biofilm growth on cellulose as a nutritive surface, primarily because it would be interesting to know the effects of biofilm formation on ethanol production, as well as on cellulose decomposition. Similar to the results of experiments with $C$. uda described above, our studies of $C$. phytofermentans biofilms indicated that cells specifically attached to nutritive surfaces. Cells tightly bound to cellulose (filter paper, cellophane, or dialysis tubing), but did not colonize abiotic surfaces such as plastic or the glass surfaces of culture vessels. 
In contrast to our findings with $C$. uda, however, C. phytofermentans biofilm formation was not regulated by nitrogen availability. Biofilm development occurred in media containing relatively high concentrations of yeast extract, which serves as a source of nitrogen for $C$. phytofermentans. In fact, under all culture conditions examined thus far, $C$. phytofermentans cells have attached to cellulose and formed microcolonies. However, addition of glucose to cultures prevented the formation of typical biofilm structures and greatly inhibited cellulose degradation. On the other hand, addition of lactose to cultures speeded the formation of robust biofilms, which was followed by rapid cellulose degradation. Addition of other sugars had a similar, but less pronounced effects on biofilm formation and cellulose degradation as addition of lactose. In most experiments, ethanol production was relatively high, exceeding acetate production. Currently, we are examining the kinetics of product formation inasmuch as some experiments have suggested an effect of growth phase on fermentation product formation.

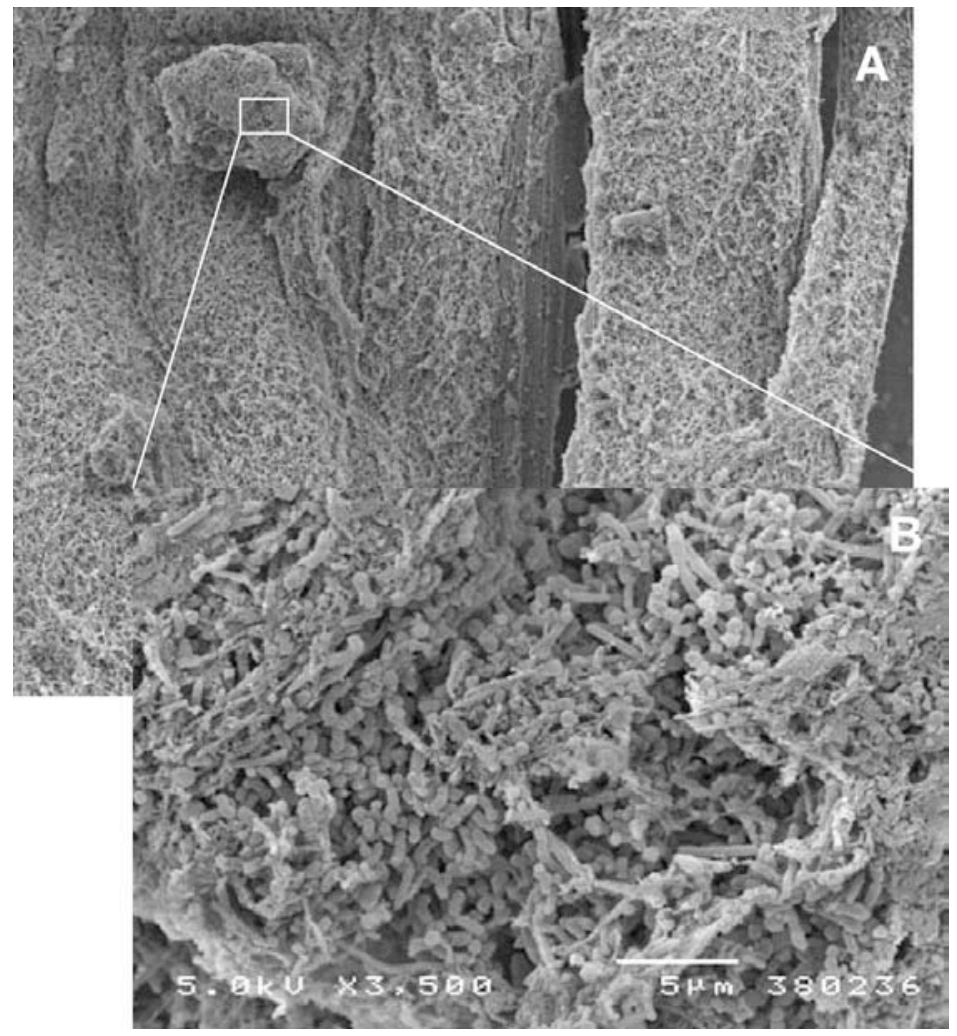

Figure 1. Scanning electron micrographs of a C. phytofermentans biofilm on dialysis tubing. A. Low magnification view of biofilm sowing a large cell aggregate on the surface of shredded dialysis tubing. B. Magnified section of the biofilm showing individual cells embedded in a stringy extracellular matrix, presumably dehydrated EPS.

C. phytofermentans biofilms on dialysis tubing were visualized by light microscopy, CSLM, and SEM, and found to have architectural features similar to those described 
above and typical of single-species biofilms (e.g., Fig. 1). Cell aggregates, embedded in an extracellular matrix, colonized the surface of the dialysis tubing in patches forming three-dimensional structures separated by open areas. These mushroom-like structures, separated by channels and irregularly shaped openings, were similar to those observed in other biofilms.

\section{Formation of biofilms by T. fusca}

$T$. fusca was cultured with dialysis tubing as a nutritive surface, or with the nonnutritive surfaces, glass, plastic, metal, and Teflon. In contrast to C. uda and C. phytofermentans, $T$. fusca cells grew as a biofilm tightly attached to both abiotic and nutritive surfaces, and in contrast to $C$. uda, nutrient limitation was not required for biofilm formation. T. fusca cells colonized all surfaces tested forming biofilms.

Dialysis tubing was colonized by $T$. fusca aleuriospores but not by mycelial pellets. Cell growth occurred as complex structures that varied in density across the surface of dialysis tubing. Scanning electron microscopy revealed cells embedded in fibrous material suggestive of an exopolymeric matrix. Concanavalin-A bound to the exopolymeric material of biofilms and mycelial pellets, indicating alpha-linked Dmannosyl and/or alpha-linked D-glucosyl residues. The carbohydrate content of both biofilms and mycelial pellets increased during growth. Also, DNase1 inhibited biofilm production suggesting a role for extracellular DNA in $T$. fusca biofilm development.

When dialysis tubing served as biofilm support, the tubing disintegrated and dissolved during incubation as a result of active degradation of the cellulosic surface. Cellulose degradation and ce/E (endoglucanase E5) expression were similar for $T$. fusca biofilms and mycelial pellets. Results of our studies indicated that, in the life cycle of this actinomycete, cellulose is specifically colonized by aleuriospores, which germinate and degrade cellulose, ultimately developing into biofilms encased in a carbohydrate-containing exopolymeric matrix, a hallmark of biofilm production. Possibly, biofilm formation on both nutritive and non-nutritive surfaces is a behavioral strategy enabling $T$. fusca cells to persist in composts and to secure and efficiently degrade insoluble cellulosic polymers when they are available.

\section{Biofilm architecture}

As mentioned above, we imaged biofilm architecture by various techniques including light microscopy, CSLM, and SEM. We also compared various staining and fixation methods in order to avoid artifacts and obtain clear and accurate images of the biofilms. Also, we used microscopy to characterize the effects of biofilm formation on the surface of the cellulose substrate. A consistent view of C. uda, C. phytofermentans, and $T$. fusca biofilm structure emerged from these studies, one that is strikingly similar to biofilm structures formed by other microbes on abiotic surfaces. Confocal images revealed a lack of homogeneity in the biofilms and side view images ( $x-y$ plane) clearly showed the variable height of cell aggregates. Cell aggregates were also clearly visualized by SEM (Fig. 1). High magnification views show extracellular material 
surrounding and interconnecting cells, some appearing string-like, presumably dehydrated EPS.

\section{Additional Investigations.}

Coculture experiments. Although we were unsuccessful in our attempts to establish stable cocultures of $C$. phytofermentans and the ethanologenic bacterium, Zymomonas mobilis, we used coculture studies as a tool to examine the nature of soluble sugars released by $C$. phytofermentans during cellulose degradation. These studies have provided new insight into the nature of the enzymatic attack on insoluble polymers by $C$. phytofermentans.

Tools for genetic manipulations. A major hindrance to our understanding of cellulose-decomposing bacteria and their application in cellulosic ethanol production has been the scarcity of methods by which genetic manipulations can be performed. One of our studies focused on C. phytofermentans. As described above, this bacterium is nutritionally versatile and capable of fermenting most major components of biomass. It also produces ethanol as its major fermentation product. Conjugation and electrotransformation were investigated as a means of introducing genetic material into C. phytofermentans cells. Transposon Tn916 was transferred from Enterococcus faecalis CG110 to Bacillus subtilis W26. The resulting transconjugant strain, B. subtilis W26T, was used as donor in filter-mating experiments to transfer Tn916 to $C$. phytofermentans. Mutant strains were selected based on resistance to tetracycline and using the $\mathrm{NaBrO}_{3}-\mathrm{NaBr}$ proton suicide technique. Strains were screened for altered motility as determined by colony morphology on agar media and for fermentation product formation using high-pressure liquid chromatography. Three transconjugates that showed altered motility were also affected in their ability to form biofilms and degrade cellulose. Electroporation was carried out with pAMß1 and pIMP1. Successful electrotransformation of $C$. phytofermentans occurred at $2.0 \mathrm{kV}$ with a resistance of $25 \mu \mathrm{fd}$ and $100 \Omega$. Transformation efficiencies (transformants/ $\mu \mathrm{g}$ DNA) were 11.4 with pAMß1and 24.0 with pIMP1. Results of these studies indicated that conjugative transposon mutagenesis and electrotransformation may be effective genetic tools for $C$. phytofermentans.

\section{PUBLICATIONS RESULTING FROM THE PROJECT}

\section{Publications based completely or in part on the work of this project.}

Warnick, T. A., and S. B. Leschine. 2002. Clostridium phytofermentans sp. nov., a cellulolytic mesophile from forest soil. International Journal of Systematic and Evolutionary Microbiology 52:1155-1160.

Reguera, G., and S. B. Leschine. 2003. Biochemical and genetic characterization of ChiA, the major enzyme component for the solubilization of chitin by Cellulomonas uda. Archives of Microbiology 180:434-443. 
Alonso, A. N., G. Reguera, and S. B. Leschine. 2003. Biofilm formation on nutritive surfaces by cellulolytic bacteria: A matter of "taste." Abstracts: Biofilms 2003 49:62.

Alonso, A. N., D. A. Bates, and S. B. Leschine. 2004. Biofilm formation by the thermophilic cellulolytic actinomycete Thermobifida fusca. Abstr. Annu. Meet. Amer. Soc. Microbiol. 104:Q216.

Alonso, A. N. and S. B. Leschine. 2004. Impacts of biofilm formation by the thermophilic cellulolytic actinomycete Thermobifida fusca, Abstracts of Papers, SACNAS National Conference, Austin, Texas.

Leschine, S. 2005. Degradation of polymers: cellulose, xylan, pectin, starch, 101-131. In P. Dürre (ed.), Handbook on Clostridia, CRC Press, Boca Raton.

Alonso, A. N., and S. B. Leschine. 2005. Exopolysaccharide production by the cellulolytic actinomycete Thermobifida fusca during biofilm development on cellulose. Abstr. Annu. Meet. Amer. Soc. Microbiol. 105:J012.

Alonso, A. N., and S. B. Leschine. 2005. Biofilms on biotic surfaces: A link in the carbon cycle on early Earth. Abstracts of Papers P-183, The $14^{\text {th }}$ International Conference on the Origin of Life, Beijing.

Alonso, A. N. and S. B. Leschine. 2006. Biofilm formation by the thermophilic cellulolytic actinomycete Thermobifida fusca, Biofilms 2: 23-24.

LaTouf, W. G., J. L.. Blanchard, and S. B. Leschine. 2006. Genomic analyses reveal loci for intracellular proteinaceous nanocompartments in Clostridium phytofermentans, a cellulolytic microbe. Abstracts of Papers, 2006 SACNAS National Conference, Tampa, Florida.

Warnick, T. A., A. H. Glickman, and S. B. Leschine. 2007. Genetic tools for the cellulolytic ethanologen Clostridium phytofermentans. Abstr. Annu. Meet. Amer. Soc. Microbiol. 107: H08.

Alonso, A. N., P. J. Pomposiello, and S. B. Leschine. 2008. Biofilm formation in the life cycle of the cellulolytic actinomycete Thermobifida fusca. Biofilms, Published online by Cambridge University Press 19 Nov 2008 doi:10.1017/S1479050508002238.

Reguera, G., Alonso, A. N., and S. B. Leschine. Biofilm formation on natural polymers by Cellulomonas uda in its quest for food. prepared for Environmental Microbiology.

Warnick, T. A., W. M. Wittbold III, D. Chirgwin, and S. B. Leschine. Colonization and degradation of cellulose by Clostridium phytofermentans biofilms, prepared for Applied and Environmental Microbiology.

Patent: Leschine, S. and T. A. Warnick. "Systems and Methods for Producing Biofuels and Related Materials." (pending) 